Supplement of Solid Earth, 10, 1301-1319, 2019

https://doi.org/10.5194/se-10-1301-2019-supplement

(C) Author(s) 2019. This work is distributed under

the Creative Commons Attribution 4.0 License.

(c) (1)

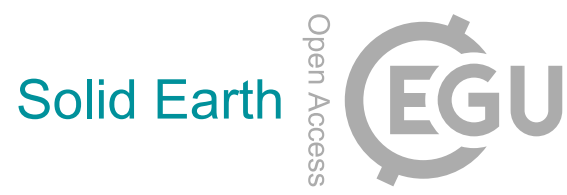

Supplement of

\title{
Monitoring of induced distributed double-couple sources using Marchenko-based virtual receivers
}

Joeri Brackenhoff et al.

Correspondence to: Joeri Brackenhoff (j.a.brackenhoff@ tudelft.nl)

The copyright of individual parts of the supplement might differ from the CC BY 4.0 License. 


\section{S1 Classical homogeneous Green's function representation}

\section{S1.1 Definition of the homogeneous Green's function}

Consider an inhomogeneous lossless acoustic medium with mass density $\rho(\boldsymbol{x})$ and compressibility $\kappa(\boldsymbol{x})$. In this medium a space- and time-dependent source distribution $q(\boldsymbol{x}, t)$ is present, with $q$ defined as the volume-injection rate density. The acoustic wave field, caused by this source distribution, is described in terms of the acoustic pressure $p(\boldsymbol{x}, t)$ and the particle velocity $v_{i}(\boldsymbol{x}, t)$. These field quantities obey the equation of motion and the stress-strain relation, according to

$$
\begin{aligned}
& \rho \partial_{t} v_{i}+\partial_{i} p=0, \\
& \kappa \partial_{t} p+\partial_{i} v_{i}=q .
\end{aligned}
$$

When $q$ is an impulsive source at $\boldsymbol{x}=\boldsymbol{x}_{\mathrm{A}}$ and $t=0$, according to

$$
q(\boldsymbol{x}, t)=\delta\left(\boldsymbol{x}-\boldsymbol{x}_{\mathrm{A}}\right) \delta(t),
$$

then the causal solution of Eqs. (S1) and (S2) defines the Green's function, hence

$$
p(\boldsymbol{x}, t)=G\left(\boldsymbol{x}, \boldsymbol{x}_{\mathrm{A}}, t\right) .
$$

By eliminating $v_{i}$ from Eqs. (S1) and (S2) and substituting Eqs. (S3) and (S4), we find that the Green's function $G\left(\boldsymbol{x}, \boldsymbol{x}_{\mathrm{A}}, t\right)$ obeys the following wave equation

$$
\partial_{i}\left(\rho^{-1} \partial_{i} G\right)-\kappa \partial_{t}^{2} G=-\delta\left(\boldsymbol{x}-\boldsymbol{x}_{\mathrm{A}}\right) \partial_{t} \delta(t) .
$$

Wave equation (S5) is symmetric in time, except for the source on the right-hand side, which is anti-symmetric. Hence, the time-reversed Green's function $G\left(\boldsymbol{x}, \boldsymbol{x}_{\mathrm{A}},-t\right)$ obeys the same wave equation, but with opposite sign for the source. By summing the wave equations for $G\left(\boldsymbol{x}, \boldsymbol{x}_{\mathrm{A}}, t\right)$ and $G\left(\boldsymbol{x}, \boldsymbol{x}_{\mathrm{A}},-t\right)$, the sources on the right-hand sides cancel each other, hence, the homogeneous Green's function

$$
G_{\mathrm{h}}\left(\boldsymbol{x}, \boldsymbol{x}_{\mathrm{A}}, t\right)=G\left(\boldsymbol{x}, \boldsymbol{x}_{\mathrm{A}}, t\right)+G\left(\boldsymbol{x}, \boldsymbol{x}_{\mathrm{A}},-t\right)
$$

obeys the homogeneous equation

$$
\partial_{i}\left(\rho^{-1} \partial_{i} G_{\mathrm{h}}\right)-\kappa \partial_{t}^{2} G_{\mathrm{h}}=0 .
$$

\section{S1.2 Reciprocity theorems}

We define the temporal Fourier transform of a time-dependent quantity $u(t)$ as

$$
u(\omega)=\int_{-\infty}^{\infty} u(t) \exp (i \omega t) \mathrm{d} t .
$$

In the frequency domain, Eqs. (S1) and (S2) transform to

$$
\begin{aligned}
& -i \omega \rho v_{i}+\partial_{i} p=0, \\
& -i \omega \kappa p+\partial_{i} v_{i}=q .
\end{aligned}
$$

We introduce two independent acoustic states, which will be distinguished by subscripts A and B. Rayleigh's reciprocity theorem is obtained by considering the quantity $\partial_{i}\left\{p_{\mathrm{A}} v_{i, B}-v_{i, A} p_{\mathrm{B}}\right\}$, applying the product rule for differentiation, substituting Eqs. (S9) and (S10) for both states, integrating the result over a spatial domain $\mathbb{V}$ enclosed by surface $\mathbb{S}$ with outward pointing 
normal $n_{i}$, and applying the theorem of Gauss (de Hoop, 1988; Fokkema and van den Berg, 1993). Assuming that in $\mathbb{V}$ the medium parameters $\rho(\boldsymbol{x})$ and $\kappa(\boldsymbol{x})$ in the two states are identical, this yields Rayleigh's reciprocity theorem of the convolution type

$$
\int_{\mathbb{V}}\left\{p_{\mathrm{A}} q_{\mathrm{B}}-q_{\mathrm{A}} p_{\mathrm{B}}\right\} \mathrm{d} \boldsymbol{x}=\oint_{\mathbb{S}} \frac{1}{i \omega \rho}\left\{p_{\mathrm{A}}\left(\partial_{i} p_{\mathrm{B}}\right)-\left(\partial_{i} p_{\mathrm{A}}\right) p_{\mathrm{B}}\right\} n_{i} \mathrm{~d} \boldsymbol{x} .
$$

We derive a second form of Rayleigh's reciprocity theorem for time-reversed wave fields. In the frequency domain, timereversal is replaced by complex conjugation. When $p$ is a solution of Eqs. (S9) and (S10) with source distribution $q$ (and real-valued medium parameters), then $p^{*}$ obeys the same equations with source distribution $-q^{*}$. Making these substitutions for state A in Eq. (S11) we obtain Rayleigh's reciprocity theorem of the correlation type (Bojarski, 1983)

$$
\int_{\mathbb{V}}\left\{p_{\mathrm{A}}^{*} q_{\mathrm{B}}+q_{\mathrm{A}}^{*} p_{\mathrm{B}}\right\} \mathrm{d} \boldsymbol{x}=\oint_{\mathbb{S}} \frac{1}{i \omega \rho}\left\{p_{\mathrm{A}}^{*}\left(\partial_{i} p_{\mathrm{B}}\right)-\left(\partial_{i} p_{\mathrm{A}}^{*}\right) p_{\mathrm{B}}\right\} n_{i} \mathrm{~d} \boldsymbol{x} .
$$

\section{S1.3 Representation of the homogeneous Green's function}

We choose point sources in both states, according to $q_{\mathrm{A}}(\boldsymbol{x}, \omega)=\delta\left(\boldsymbol{x}-\boldsymbol{x}_{\mathrm{A}}\right)$ and $q_{\mathrm{B}}(\boldsymbol{x}, \omega)=\delta\left(\boldsymbol{x}-\boldsymbol{x}_{\mathrm{B}}\right)$, with $\boldsymbol{x}_{\mathrm{A}}$ and $\boldsymbol{x}_{\mathrm{B}}$ both in $\mathbb{V}$. The fields in states A and B are thus expressed in terms of Green's functions, according to

$$
\begin{aligned}
& p_{\mathrm{A}}(\boldsymbol{x}, \omega)=G\left(\boldsymbol{x}, \boldsymbol{x}_{\mathrm{A}}, \omega\right), \\
& p_{\mathrm{B}}(\boldsymbol{x}, \omega)=G\left(\boldsymbol{x}, \boldsymbol{x}_{\mathrm{B}}, \omega\right),
\end{aligned}
$$

with $G\left(\boldsymbol{x}, \boldsymbol{x}_{\mathrm{A}}, \omega\right)$ and $G\left(\boldsymbol{x}, \boldsymbol{x}_{\mathrm{B}}, \omega\right)$ being the Fourier transforms of $G\left(\boldsymbol{x}, \boldsymbol{x}_{\mathrm{A}}, t\right)$ and $G\left(\boldsymbol{x}, \boldsymbol{x}_{\mathrm{B}}, t\right)$, respectively. Making these substitutions in Eq. (S12) and using source-receiver reciprocity of the Green's functions gives (Porter, 1970; Oristaglio, 1989; Wapenaar, 2004; Van Manen et al., 2005)

$$
G_{\mathrm{h}}\left(\boldsymbol{x}_{\mathrm{B}}, \boldsymbol{x}_{\mathrm{A}}, \omega\right)=\oint_{\mathbb{S}} \frac{1}{i \omega \rho(\boldsymbol{x})}\left(\left\{\partial_{i} G\left(\boldsymbol{x}, \boldsymbol{x}_{\mathrm{B}}, \omega\right)\right\} G^{*}\left(\boldsymbol{x}, \boldsymbol{x}_{\mathrm{A}}, \omega\right)-G\left(\boldsymbol{x}, \boldsymbol{x}_{\mathrm{B}}, \omega\right) \partial_{i} G^{*}\left(\boldsymbol{x}, \boldsymbol{x}_{\mathrm{A}}, \omega\right)\right) n_{i} \mathrm{~d} \boldsymbol{x},
$$

where $G_{\mathrm{h}}\left(\boldsymbol{x}_{\mathrm{B}}, \boldsymbol{x}_{\mathrm{A}}, \omega\right)$ is the homogeneous Green's function in the frequency domain. It is defined as

$$
G_{\mathrm{h}}\left(\boldsymbol{x}, \boldsymbol{x}_{\mathrm{A}}, \omega\right)=G\left(\boldsymbol{x}, \boldsymbol{x}_{\mathrm{A}}, \omega\right)+G^{*}\left(\boldsymbol{x}, \boldsymbol{x}_{\mathrm{A}}, \omega\right)=2 \Re\left\{G\left(\boldsymbol{x}, \boldsymbol{x}_{\mathrm{A}}, \omega\right)\right\},
$$

where $\Re$ denotes the real part. Equation (S15) is an exact representation for the homogeneous Green's function $G_{\mathrm{h}}\left(\boldsymbol{x}_{\mathrm{B}}, \boldsymbol{x}_{\mathrm{A}}, \omega\right)$.

When $\mathbb{S}$ is sufficiently smooth and the medium outside $\mathbb{S}$ is homogeneous (with mass density $\rho_{0}$, compressibility $\kappa_{0}$ and propagation velocity $c_{0}=\left(\kappa_{0} \rho_{0}\right)^{-1 / 2}$ ), the two terms under the integral in Eq. (S15) are nearly identical (but opposite in sign), hence

$$
G_{\mathrm{h}}\left(\boldsymbol{x}_{\mathrm{B}}, \boldsymbol{x}_{\mathrm{A}}, \omega\right)=-2 \oint_{\mathbb{S}} \frac{1}{i \omega \rho_{0}} G\left(\boldsymbol{x}, \boldsymbol{x}_{\mathrm{B}}, \omega\right) \partial_{i} G^{*}\left(\boldsymbol{x}, \boldsymbol{x}_{\mathrm{A}}, \omega\right) n_{i} \mathrm{~d} \boldsymbol{x} .
$$

The main approximation is that evanescent waves are neglected at $\mathbb{S}$ (Zheng et al., 2011; Wapenaar et al., 2011).

\section{S2 Single-sided homogeneous Green's function representations}

\section{S2.1 Modification of the configuration}

We replace the arbitrary closed surface $\mathbb{S}$ by a combination of two surfaces $\mathbb{S}_{0}$ and $\mathbb{S}_{\mathrm{A}}$, as indicated in Fig. S1. Here $\mathbb{S}_{0}$ may be curved, but $\mathbb{S}_{\mathrm{A}}$ is a horizontal surface, with $\boldsymbol{n}=(0,0,1)$. The depth level of $\mathbb{S}_{\mathrm{A}}$ is defined as $x_{3, \mathrm{~A}}$ (which is equal to 


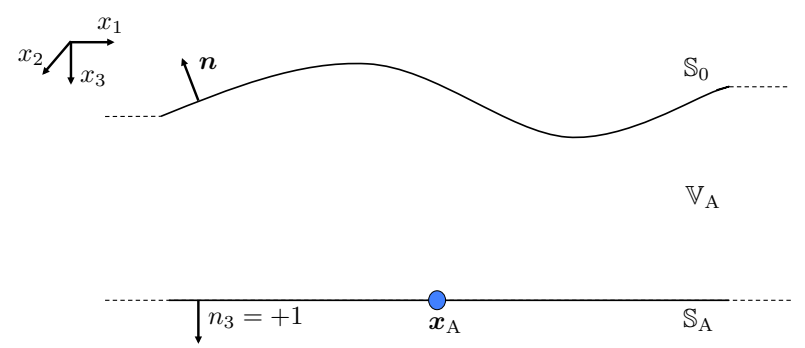

Figure S1. Modified configuration. The surface $\mathbb{S}$ consists of the combination of surfaces $\mathbb{S}_{0}$ and $\mathbb{S}_{\mathrm{A}}$.

the $x_{3}$-coordinate of the point $\left.\boldsymbol{x}_{\mathrm{A}}\right)$. The domain between surfaces $\mathbb{S}_{0}$ and $\mathbb{S}_{\mathrm{A}}$ is called $\mathbb{V}_{\mathrm{A}}$. For this configuration, reciprocity theorems (S11) and (S12) are replaced by

$$
\int_{\mathbb{V}_{\mathrm{A}}}\left\{p_{\mathrm{A}} q_{\mathrm{B}}-q_{\mathrm{A}} p_{\mathrm{B}}\right\} \mathrm{d} \boldsymbol{x}=\int_{\mathbb{S}_{0}} \frac{1}{i \omega \rho}\left\{p_{\mathrm{A}}\left(\partial_{i} p_{\mathrm{B}}\right)-\left(\partial_{i} p_{\mathrm{A}}\right) p_{\mathrm{B}}\right\} n_{i} \mathrm{~d} \boldsymbol{x}+\int_{\mathbb{S}_{\mathrm{A}}} \frac{1}{i \omega \rho}\left\{p_{\mathrm{A}}\left(\partial_{3} p_{\mathrm{B}}\right)-\left(\partial_{3} p_{\mathrm{A}}\right) p_{\mathrm{B}}\right\} \mathrm{d} \boldsymbol{x}
$$

and

$$
\int_{\mathbb{V}_{\mathrm{A}}}\left\{p_{\mathrm{A}}^{*} q_{\mathrm{B}}+q_{\mathrm{A}}^{*} p_{\mathrm{B}}\right\} \mathrm{d} \boldsymbol{x}=\int_{\mathbb{S}_{0}} \frac{1}{i \omega \rho}\left\{p_{\mathrm{A}}^{*}\left(\partial_{i} p_{\mathrm{B}}\right)-\left(\partial_{i} p_{\mathrm{A}}^{*}\right) p_{\mathrm{B}}\right\} n_{i} \mathrm{~d} \boldsymbol{x}+\int_{\mathbb{S}_{\mathrm{A}}} \frac{1}{i \omega \rho}\left\{p_{\mathrm{A}}^{*}\left(\partial_{3} p_{\mathrm{B}}\right)-\left(\partial_{3} p_{\mathrm{A}}^{*}\right) p_{\mathrm{B}}\right\} \mathrm{d} \boldsymbol{x},
$$

respectively. In the following we use these reciprocity theorems as the basis for deriving several versions of single-sided homogeneous Green's function representations, each time by applying decomposition to one or more of the integrals in these theorems.

\section{S2.2 Single-sided homogeneous Green's function representation: general formulation}

Following a similar derivation as in Appendix B in Wapenaar and Berkhout (1989), we reformulate Eqs. (S18) and (S19) as

$$
\int_{\mathbb{V}_{\mathrm{A}}}\left(p_{\mathrm{A}} q_{\mathrm{B}}-q_{\mathrm{A}} p_{\mathrm{B}}\right) \mathrm{d} \boldsymbol{x}=\int_{\mathbb{S}_{0}} \frac{1}{i \omega \rho}\left(p_{\mathrm{A}}\left(\partial_{i} p_{\mathrm{B}}\right)-\left(\partial_{i} p_{\mathrm{A}}\right) p_{\mathrm{B}}\right) n_{i} \mathrm{~d} \boldsymbol{x}-\int_{\mathbb{S}_{\mathrm{A}}} \frac{2}{i \omega \rho}\left(\left(\partial_{3} p_{\mathrm{A}}^{+}\right) p_{\mathrm{B}}^{-}+\left(\partial_{3} p_{\mathrm{A}}^{-}\right) p_{\mathrm{B}}^{+}\right) \mathrm{d} \boldsymbol{x}
$$

and, ignoring evanescent waves,

$$
\int_{\mathbb{V}_{\mathrm{A}}}\left(p_{\mathrm{A}}^{*} q_{\mathrm{B}}+q_{\mathrm{A}}^{*} p_{\mathrm{B}}\right) \mathrm{d} \boldsymbol{x}=\int_{\mathbb{S}_{0}} \frac{1}{i \omega \rho}\left(p_{\mathrm{A}}^{*}\left(\partial_{i} p_{\mathrm{B}}\right)-\left(\partial_{i} p_{\mathrm{A}}^{*}\right) p_{\mathrm{B}}\right) n_{i} \mathrm{~d} \boldsymbol{x}-\int_{\mathbb{S}_{\mathrm{A}}} \frac{2}{i \omega \rho}\left(\left(\partial_{3} p_{\mathrm{A}}^{+}\right)^{*} p_{\mathrm{B}}^{+}+\left(\partial_{3} p_{\mathrm{A}}^{-}\right)^{*} p_{\mathrm{B}}^{-}\right) \mathrm{d} \boldsymbol{x} .
$$

The superscripts + and - stand for downgoing and upgoing, respectively. For state A we consider the focusing function $f_{1}\left(\boldsymbol{x}, \boldsymbol{x}_{\mathrm{A}}, \omega\right)=f_{1}^{+}\left(\boldsymbol{x}, \boldsymbol{x}_{\mathrm{A}}, \omega\right)+f_{1}^{-}\left(\boldsymbol{x}, \boldsymbol{x}_{\mathrm{A}}, \omega\right)$, introduced in section 3.1 in the companion paper "Green's theorem in seismic imaging across the scales" (Wapenaar et al., 2019). This focusing function is defined in a truncated version of the medium, which is identical to the actual medium in $\mathbb{V}_{\mathrm{A}}$, but reflection free above $\mathbb{S}_{0}$ and below $\mathbb{S}_{\mathrm{A}}$. The focusing conditions at the focal plane $\mathbb{S}_{\mathrm{A}}$ are (Wapenaar et al., 2014)

$$
\begin{aligned}
& {\left[\partial_{3} f_{1}^{+}\left(\boldsymbol{x}, \boldsymbol{x}_{\mathrm{A}}, \omega\right)\right]_{x_{3}=x_{3, \mathrm{~A}}}=\frac{1}{2} i \omega \rho\left(\boldsymbol{x}_{\mathrm{A}}\right) \delta\left(\boldsymbol{x}_{\mathrm{H}}-\boldsymbol{x}_{\mathrm{H}, \mathrm{A}}\right),} \\
& {\left[\partial_{3} f_{1}^{-}\left(\boldsymbol{x}, \boldsymbol{x}_{\mathrm{A}}, \omega\right)\right]_{x_{3}=x_{3, \mathrm{~A}}}=0 .}
\end{aligned}
$$

For state B we consider the Green's function $G\left(\boldsymbol{x}, \boldsymbol{x}_{\mathrm{B}}, \omega\right)=G^{+}\left(\boldsymbol{x}, \boldsymbol{x}_{\mathrm{B}}, \omega\right)+G^{-}\left(\boldsymbol{x}, \boldsymbol{x}_{\mathrm{B}}, \omega\right)$, with its source at $\boldsymbol{x}_{\mathrm{B}}$ anywhere in the half-space below $\mathbb{S}_{0}$. Note that the superscripts + and - in $f_{1}^{ \pm}\left(\boldsymbol{x}, \boldsymbol{x}_{\mathrm{A}}, \omega\right)$ and $G^{ \pm}\left(\boldsymbol{x}, \boldsymbol{x}_{\mathrm{B}}, \omega\right)$ refer to the propagation 
direction (downward or upward) at the observation point $\boldsymbol{x}$. The source of the Green's function at $\boldsymbol{x}_{\mathrm{B}}$ is omnidirectional. Substituting $q_{\mathrm{A}}(\boldsymbol{x}, \omega)=0, p_{\mathrm{A}}^{ \pm}(\boldsymbol{x}, \omega)=f_{1}^{ \pm}\left(\boldsymbol{x}, \boldsymbol{x}_{\mathrm{A}}, \omega\right), q_{\mathrm{B}}(\boldsymbol{x}, \omega)=\delta\left(\boldsymbol{x}-\boldsymbol{x}_{\mathrm{B}}\right)$ and $p_{\mathrm{B}}^{ \pm}(\boldsymbol{x}, \omega)=G^{ \pm}\left(\boldsymbol{x}, \boldsymbol{x}_{\mathrm{B}}, \omega\right)$ into Eqs. (S20) and (S21), using Eqs. (S22) and (S23), gives

$$
\begin{aligned}
G^{-} & \left(\boldsymbol{x}_{\mathrm{A}}, \boldsymbol{x}_{\mathrm{B}}, \omega\right)+\chi\left(\boldsymbol{x}_{\mathrm{B}}\right) f_{1}\left(\boldsymbol{x}_{\mathrm{B}}, \boldsymbol{x}_{\mathrm{A}}, \omega\right) \\
\quad= & \int_{\mathbb{S}_{0}} \frac{1}{i \omega \rho(\boldsymbol{x})}\left(\left\{\partial_{i} G\left(\boldsymbol{x}, \boldsymbol{x}_{\mathrm{B}}, \omega\right)\right\} f_{1}\left(\boldsymbol{x}, \boldsymbol{x}_{\mathrm{A}}, \omega\right)-G\left(\boldsymbol{x}, \boldsymbol{x}_{\mathrm{B}}, \omega\right) \partial_{i} f_{1}\left(\boldsymbol{x}, \boldsymbol{x}_{\mathrm{A}}, \omega\right)\right) n_{i} \mathrm{~d} \boldsymbol{x}
\end{aligned}
$$

and

$$
\begin{aligned}
& G^{+}\left(\boldsymbol{x}_{\mathrm{A}}, \boldsymbol{x}_{\mathrm{B}}, \omega\right)-\chi\left(\boldsymbol{x}_{\mathrm{B}}\right) f_{1}^{*}\left(\boldsymbol{x}_{\mathrm{B}}, \boldsymbol{x}_{\mathrm{A}}, \omega\right) \\
& \quad=-\int_{\mathbb{S}_{0}} \frac{1}{i \omega \rho(\boldsymbol{x})}\left(\left\{\partial_{i} G\left(\boldsymbol{x}, \boldsymbol{x}_{\mathrm{B}}, \omega\right)\right\} f_{1}^{*}\left(\boldsymbol{x}, \boldsymbol{x}_{\mathrm{A}}, \omega\right)-G\left(\boldsymbol{x}, \boldsymbol{x}_{\mathrm{B}}, \omega\right) \partial_{i} f_{1}^{*}\left(\boldsymbol{x}, \boldsymbol{x}_{\mathrm{A}}, \omega\right)\right) n_{i} \mathrm{~d} \boldsymbol{x},
\end{aligned}
$$

respectively, where $\chi$ is the characteristic function of the domain $\mathbb{V}_{\mathrm{A}}$. It is defined as

$$
\chi\left(\boldsymbol{x}_{\mathrm{B}}\right)= \begin{cases}1, & \text { for } \boldsymbol{x}_{\mathrm{B}} \text { between } \mathbb{S}_{0} \text { and } \mathbb{S}_{\mathrm{A}}, \\ \frac{1}{2}, & \text { for } \boldsymbol{x}_{\mathrm{B}} \text { on } \mathbb{S}=\mathbb{S}_{0} \cup \mathbb{S}_{\mathrm{A}} \\ 0, & \text { for } \boldsymbol{x}_{\mathrm{B}} \text { outside } \mathbb{S} .\end{cases}
$$

Summing Eqs. (S24) and (S25) and using source-receiver reciprocity for the Green's function on the left-hand side yields

$$
\begin{aligned}
& G\left(\boldsymbol{x}_{\mathrm{B}}, \boldsymbol{x}_{\mathrm{A}}, \omega\right)+\chi\left(\boldsymbol{x}_{\mathrm{B}}\right) 2 i \Im\left\{f_{1}\left(\boldsymbol{x}_{\mathrm{B}}, \boldsymbol{x}_{\mathrm{A}}, \omega\right)\right\} \\
& \quad=\int_{\mathbb{S}_{0}} \frac{2}{\omega \rho(\boldsymbol{x})}\left(\left\{\partial_{i} G\left(\boldsymbol{x}, \boldsymbol{x}_{\mathrm{B}}, \omega\right)\right\} \Im\left\{f_{1}\left(\boldsymbol{x}, \boldsymbol{x}_{\mathrm{A}}, \omega\right)\right\}-G\left(\boldsymbol{x}, \boldsymbol{x}_{\mathrm{B}}, \omega\right) \Im\left\{\partial_{i} f_{1}\left(\boldsymbol{x}, \boldsymbol{x}_{\mathrm{A}}, \omega\right)\right\}\right) n_{i} \mathrm{~d} \boldsymbol{x},
\end{aligned}
$$

where $\Im$ denotes the imaginary part. Taking the real part of both sides of this equation, using Eq. (S16), gives the single-sided representation of the homogeneous Green's function

$$
G_{\mathrm{h}}\left(\boldsymbol{x}_{\mathrm{B}}, \boldsymbol{x}_{\mathrm{A}}, \omega\right)=\int_{\mathbb{S}_{0}} \frac{2}{\omega \rho(\boldsymbol{x})}\left(\left\{\partial_{i} G_{\mathrm{h}}\left(\boldsymbol{x}, \boldsymbol{x}_{\mathrm{B}}, \omega\right)\right\} \Im\left\{f_{1}\left(\boldsymbol{x}, \boldsymbol{x}_{\mathrm{A}}, \omega\right)\right\}-G_{\mathrm{h}}\left(\boldsymbol{x}, \boldsymbol{x}_{\mathrm{B}}, \omega\right) \Im\left\{\partial_{i} f_{1}\left(\boldsymbol{x}, \boldsymbol{x}_{\mathrm{A}}, \omega\right)\right\}\right) n_{i} \mathrm{~d} \boldsymbol{x} .
$$

\section{S2.3 Single-sided homogeneous Green's function representation: assuming a homogeneous upper half-space}

From here onward we assume that also $\mathbb{S}_{0}$ is a horizontal surface, with $\boldsymbol{n}=(0,0,-1)$. Following a similar derivation as in Appendix B in Wapenaar and Berkhout (1989), we reformulate Eqs. (S18) and (S19) as

$$
\begin{aligned}
\int_{\mathbb{V}_{\mathrm{A}}}\left(p_{\mathrm{A}}^{+} q_{\mathrm{B}}^{-}+p_{\mathrm{A}}^{-} q_{\mathrm{B}}^{+}-q_{\mathrm{A}}^{+} p_{\mathrm{B}}^{-}-q_{\mathrm{A}}^{-} p_{\mathrm{B}}^{+}\right) \mathrm{d} \boldsymbol{x}= \\
\qquad \int_{\mathbb{S}_{0}} \frac{2}{i \omega \rho}\left(\left(\partial_{3} p_{\mathrm{A}}^{+}\right) p_{\mathrm{B}}^{-}+\left(\partial_{3} p_{\mathrm{A}}^{-}\right) p_{\mathrm{B}}^{+}\right) \mathrm{d} \boldsymbol{x}-\int_{\mathbb{S}_{\mathrm{A}}} \frac{2}{i \omega \rho}\left(\left(\partial_{3} p_{\mathrm{A}}^{+}\right) p_{\mathrm{B}}^{-}+\left(\partial_{3} p_{\mathrm{A}}^{-}\right) p_{\mathrm{B}}^{+}\right) \mathrm{d} \boldsymbol{x}
\end{aligned}
$$

and, ignoring evanescent waves,

$$
\begin{aligned}
\int_{\mathbb{V}_{\mathrm{A}}}\left(p_{\mathrm{A}}^{+*} q_{\mathrm{B}}^{+}+p_{\mathrm{A}}^{-*} q_{\mathrm{B}}^{-}+q_{\mathrm{A}}^{+*} p_{\mathrm{B}}^{+}+q_{\mathrm{A}}^{-*} p_{\mathrm{B}}^{-}\right) \mathrm{d} \boldsymbol{x} & = \\
& \int_{\mathbb{S}_{0}} \frac{2}{i \omega \rho}\left(\left(\partial_{3} p_{\mathrm{A}}^{+}\right)^{*} p_{\mathrm{B}}^{+}+\left(\partial_{3} p_{\mathrm{A}}^{-}\right)^{*} p_{\mathrm{B}}^{-}\right) \mathrm{d} \boldsymbol{x}-\int_{\mathbb{S}_{\mathrm{A}}} \frac{2}{i \omega \rho}\left(\left(\partial_{3} p_{\mathrm{A}}^{+}\right)^{*} p_{\mathrm{B}}^{+}+\left(\partial_{3} p_{\mathrm{A}}^{-}\right)^{*} p_{\mathrm{B}}^{-}\right) \mathrm{d} \boldsymbol{x} .
\end{aligned}
$$


We apply these theorems to the situation in which the upper half-space above $\mathbb{S}_{0}$ is homogeneous (for the Green's function as well as for the focusing function). For state A we consider again the focusing function $f_{1}\left(\boldsymbol{x}, \boldsymbol{x}_{\mathrm{A}}, \omega\right)=f_{1}^{+}\left(\boldsymbol{x}, \boldsymbol{x}_{\mathrm{A}}, \omega\right)+$ $f_{1}^{-}\left(\boldsymbol{x}, \boldsymbol{x}_{\mathrm{A}}, \omega\right)$, defined in a truncated version of the medium. For state B we consider the Green's function $G\left(\boldsymbol{x}, \boldsymbol{x}_{\mathrm{B}}, \omega\right)=$ $G^{+,+}\left(\boldsymbol{x}, \boldsymbol{x}_{\mathrm{B}}, \omega\right)+G^{-,+}\left(\boldsymbol{x}, \boldsymbol{x}_{\mathrm{B}}, \omega\right)+G^{+,-}\left(\boldsymbol{x}, \boldsymbol{x}_{\mathrm{B}}, \omega\right)+G^{-,-}\left(\boldsymbol{x}, \boldsymbol{x}_{\mathrm{B}}, \omega\right)$, with its source at $\boldsymbol{x}_{\mathrm{B}}$ anywhere in the half-space below $\mathbb{S}_{0}$. Note that we introduced two superscripts. The first superscript refers again to the propagation direction at the observation point $\boldsymbol{x}$. The second superscript refers to the radiation direction of the source at $\boldsymbol{x}_{\mathrm{B}}$. Substituting $q_{\mathrm{A}}^{+}(\boldsymbol{x}, \omega)=q_{\mathrm{A}}^{-}(\boldsymbol{x}, \omega)=0$, $p_{\mathrm{A}}^{ \pm}(\boldsymbol{x}, \omega)=f_{1}^{ \pm}\left(\boldsymbol{x}, \boldsymbol{x}_{\mathrm{A}}, \omega\right), q_{\mathrm{B}}^{+}(\boldsymbol{x}, \omega)=\delta\left(\boldsymbol{x}-\boldsymbol{x}_{\mathrm{B}}\right), q_{\mathrm{B}}^{-}(\boldsymbol{x}, \omega)=0$ and $p_{\mathrm{B}}^{ \pm}(\boldsymbol{x}, \omega)=G^{ \pm,+}\left(\boldsymbol{x}, \boldsymbol{x}_{\mathrm{B}}, \omega\right)$ into Eqs. (S29) and (S30), using Eqs. (S22) and (S23) and $G^{+,+}\left(\boldsymbol{x}, \boldsymbol{x}_{\mathrm{B}}, \omega\right)=0$ for $\boldsymbol{x}$ at $\mathbb{S}_{0}$ (since the upper half-space is homogeneous), gives

$$
G^{-,+}\left(\boldsymbol{x}_{\mathrm{A}}, \boldsymbol{x}_{\mathrm{B}}, \omega\right)+\chi\left(\boldsymbol{x}_{\mathrm{B}}\right) f_{1}^{-}\left(\boldsymbol{x}_{\mathrm{B}}, \boldsymbol{x}_{\mathrm{A}}, \omega\right)=\int_{\mathbb{S}_{0}} \frac{2}{i \omega \rho_{0}} G^{-,+}\left(\boldsymbol{x}, \boldsymbol{x}_{\mathrm{B}}, \omega\right) \partial_{3} f_{1}^{+}\left(\boldsymbol{x}, \boldsymbol{x}_{\mathrm{A}}, \omega\right) \mathrm{d} \boldsymbol{x}
$$

and

$$
G^{+,+}\left(\boldsymbol{x}_{\mathrm{A}}, \boldsymbol{x}_{\mathrm{B}}, \omega\right)-\chi\left(\boldsymbol{x}_{\mathrm{B}}\right)\left\{f_{1}^{+}\left(\boldsymbol{x}_{\mathrm{B}}, \boldsymbol{x}_{\mathrm{A}}, \omega\right)\right\}^{*}=-\int_{\mathbb{S}_{0}} \frac{2}{i \omega \rho_{0}} G^{-,+}\left(\boldsymbol{x}, \boldsymbol{x}_{\mathrm{B}}, \omega\right)\left\{\partial_{3} f_{1}^{-}\left(\boldsymbol{x}, \boldsymbol{x}_{\mathrm{A}}, \omega\right)\right\}^{*} \mathrm{~d} \boldsymbol{x} .
$$

Next, substituting $q_{\mathrm{A}}^{+}(\boldsymbol{x}, \omega)=q_{\mathrm{A}}^{-}(\boldsymbol{x}, \omega)=0, p_{\mathrm{A}}^{ \pm}(\boldsymbol{x}, \omega)=f_{1}^{ \pm}\left(\boldsymbol{x}, \boldsymbol{x}_{\mathrm{A}}, \omega\right), q_{\mathrm{B}}^{+}(\boldsymbol{x}, \omega)=0, q_{\mathrm{B}}^{-}(\boldsymbol{x}, \omega)=\delta\left(\boldsymbol{x}-\boldsymbol{x}_{\mathrm{B}}\right)$ and $p_{\mathrm{B}}^{ \pm}(\boldsymbol{x}, \omega)=$ $G^{ \pm,-}\left(\boldsymbol{x}, \boldsymbol{x}_{\mathrm{B}}, \omega\right)$ into Eqs. (S29) and (S30), using Eqs. (S22) and (S23) and $G^{+,-}\left(\boldsymbol{x}, \boldsymbol{x}_{\mathrm{B}}, \omega\right)=0$ for $\boldsymbol{x}$ at $\mathbb{S}_{0}$, gives

$$
G^{-,-}\left(\boldsymbol{x}_{\mathrm{A}}, \boldsymbol{x}_{\mathrm{B}}, \omega\right)+\chi\left(\boldsymbol{x}_{\mathrm{B}}\right) f_{1}^{+}\left(\boldsymbol{x}_{\mathrm{B}}, \boldsymbol{x}_{\mathrm{A}}, \omega\right)=\int_{\mathbb{S}_{0}} \frac{2}{i \omega \rho_{0}} G^{-,-}\left(\boldsymbol{x}, \boldsymbol{x}_{\mathrm{B}}, \omega\right) \partial_{3} f_{1}^{+}\left(\boldsymbol{x}, \boldsymbol{x}_{\mathrm{A}}, \omega\right) \mathrm{d} \boldsymbol{x}
$$

and

$$
G^{+,-}\left(\boldsymbol{x}_{\mathrm{A}}, \boldsymbol{x}_{\mathrm{B}}, \omega\right)-\chi\left(\boldsymbol{x}_{\mathrm{B}}\right)\left\{f_{1}^{-}\left(\boldsymbol{x}_{\mathrm{B}}, \boldsymbol{x}_{\mathrm{A}}, \omega\right)\right\}^{*}=-\int_{\mathbb{S}_{0}} \frac{2}{i \omega \rho_{0}} G^{-,-}\left(\boldsymbol{x}, \boldsymbol{x}_{\mathrm{B}}, \omega\right)\left\{\partial_{3} f_{1}^{-}\left(\boldsymbol{x}, \boldsymbol{x}_{\mathrm{A}}, \omega\right)\right\}^{*} \mathrm{~d} \boldsymbol{x} .
$$

Summing Eqs. (S31) - (S34), using source-receiver reciprocity for the Green's function on the left-hand side and $G^{+,+}\left(\boldsymbol{x}, \boldsymbol{x}_{\mathrm{B}}, \omega\right)=G^{+,-}\left(\boldsymbol{x}, \boldsymbol{x}_{\mathrm{B}}, \omega\right)=0$ for $\boldsymbol{x}$ at $\mathbb{S}_{0}$, we obtain

$$
\begin{aligned}
G\left(\boldsymbol{x}_{\mathrm{B}}, \boldsymbol{x}_{\mathrm{A}}, \omega\right)+\chi\left(\boldsymbol{x}_{\mathrm{B}}\right) 2 i \Im\left\{f_{1}\left(\boldsymbol{x}_{\mathrm{B}}, \boldsymbol{x}_{\mathrm{A}}, \omega\right)\right\} \\
\quad=\int_{\mathbb{S}_{0}} \frac{2}{i \omega \rho_{0}} G\left(\boldsymbol{x}, \boldsymbol{x}_{\mathrm{B}}, \omega\right) \partial_{3}\left(f_{1}^{+}\left(\boldsymbol{x}, \boldsymbol{x}_{\mathrm{A}}, \omega\right)-\left\{f_{1}^{-}\left(\boldsymbol{x}, \boldsymbol{x}_{\mathrm{A}}, \omega\right)\right\}^{*}\right) \mathrm{d} \boldsymbol{x} .
\end{aligned}
$$

Taking the real part of both sides gives the single-sided representation of the homogeneous Green's function for the situation that the upper half-space is homogeneous

$$
G_{\mathrm{h}}\left(\boldsymbol{x}_{\mathrm{B}}, \boldsymbol{x}_{\mathrm{A}}, \omega\right)=4 \Re \int_{\mathbb{S}_{0}} \frac{1}{i \omega \rho_{0}} G\left(\boldsymbol{x}, \boldsymbol{x}_{\mathrm{B}}, \omega\right) \partial_{3}\left(f_{1}^{+}\left(\boldsymbol{x}, \boldsymbol{x}_{\mathrm{A}}, \omega\right)-\left\{f_{1}^{-}\left(\boldsymbol{x}, \boldsymbol{x}_{\mathrm{A}}, \omega\right)\right\}^{*}\right) \mathrm{d} \boldsymbol{x} .
$$

We conclude by deriving source-receiver reciprocity relations for the decomposed Green's functions $G^{ \pm, \pm}\left(\boldsymbol{x}, \boldsymbol{x}_{\mathrm{B}}, \omega\right)$. We consider Eq. (S29), but replace $\mathbb{V}_{\mathrm{A}}$ by the entire space $\mathbb{R}^{3}$. In this situation there are only outgoing waves at $\mathbb{S}$. Hence, Eq. (S29) simplifies to

$$
\int_{\mathbb{R}^{3}}\left(p_{\mathrm{A}}^{+} q_{\mathrm{B}}^{-}+p_{\mathrm{A}}^{-} q_{\mathrm{B}}^{+}-q_{\mathrm{A}}^{+} p_{\mathrm{B}}^{-}-q_{\mathrm{A}}^{-} p_{\mathrm{B}}^{+}\right) \mathrm{d} \boldsymbol{x}=0 .
$$

First we substitute $q_{\mathrm{A}}^{+}=\delta\left(\boldsymbol{x}-\boldsymbol{x}_{\mathrm{A}}\right), q_{\mathrm{A}}^{-}=0, p_{\mathrm{A}}^{ \pm}=G^{ \pm,+}\left(\boldsymbol{x}, \boldsymbol{x}_{\mathrm{A}}, \omega\right), q_{\mathrm{B}}^{+}=\delta\left(\boldsymbol{x}-\boldsymbol{x}_{\mathrm{B}}\right), q_{\mathrm{B}}^{-}=0$ and $p_{\mathrm{B}}^{ \pm}=G^{ \pm,+}\left(\boldsymbol{x}, \boldsymbol{x}_{\mathrm{B}}, \omega\right)$. This gives

$$
G^{-,+}\left(\boldsymbol{x}_{\mathrm{B}}, \boldsymbol{x}_{\mathrm{A}}, \omega\right)=G^{-,+}\left(\boldsymbol{x}_{\mathrm{A}}, \boldsymbol{x}_{\mathrm{B}}, \omega\right) .
$$


Next, we substitute $q_{\mathrm{A}}^{+}=\delta\left(\boldsymbol{x}-\boldsymbol{x}_{\mathrm{A}}\right), q_{\mathrm{A}}^{-}=0, p_{\mathrm{A}}^{ \pm}=G^{ \pm,+}\left(\boldsymbol{x}, \boldsymbol{x}_{\mathrm{A}}, \omega\right), q_{\mathrm{B}}^{+}=0, q_{\mathrm{B}}^{-}=\delta\left(\boldsymbol{x}-\boldsymbol{x}_{\mathrm{B}}\right)$ and $p_{\mathrm{B}}^{ \pm}=G^{ \pm,-}\left(\boldsymbol{x}, \boldsymbol{x}_{\mathrm{B}}, \omega\right)$. This gives

$$
G^{+,+}\left(\boldsymbol{x}_{\mathrm{B}}, \boldsymbol{x}_{\mathrm{A}}, \omega\right)=G^{-,-}\left(\boldsymbol{x}_{\mathrm{A}}, \boldsymbol{x}_{\mathrm{B}}, \omega\right) .
$$

Finally, we substitute $q_{\mathrm{A}}^{+}=0, q_{\mathrm{A}}^{-}=\delta\left(\boldsymbol{x}-\boldsymbol{x}_{\mathrm{A}}\right), p_{\mathrm{A}}^{ \pm}=G^{ \pm,-}\left(\boldsymbol{x}, \boldsymbol{x}_{\mathrm{A}}, \omega\right), q_{\mathrm{B}}^{+}=0, q_{\mathrm{B}}^{-}=\delta\left(\boldsymbol{x}-\boldsymbol{x}_{\mathrm{B}}\right)$ and $p_{\mathrm{B}}^{ \pm}=G^{ \pm,-}\left(\boldsymbol{x}, \boldsymbol{x}_{\mathrm{B}}, \omega\right)$. This gives

$$
G^{+,-}\left(\boldsymbol{x}_{\mathrm{B}}, \boldsymbol{x}_{\mathrm{A}}, \omega\right)=G^{+,-}\left(\boldsymbol{x}_{\mathrm{A}}, \boldsymbol{x}_{\mathrm{B}}, \omega\right) .
$$

Note that Eq. (S39) does not include a minus sign, unlike the corresponding relation for the flux-normalised decomposed Green's functions (Wapenaar, 1996). As a result of this definition, we have the following simple expression for the full Green's function

$$
G\left(\boldsymbol{x}, \boldsymbol{x}_{\mathrm{A}}, \omega\right)=G^{+,+}\left(\boldsymbol{x}, \boldsymbol{x}_{\mathrm{A}}, \omega\right)+G^{-,+}\left(\boldsymbol{x}, \boldsymbol{x}_{\mathrm{A}}, \omega\right)+G^{+,-}\left(\boldsymbol{x}, \boldsymbol{x}_{\mathrm{A}}, \omega\right)+G^{-,-}\left(\boldsymbol{x}, \boldsymbol{x}_{\mathrm{A}}, \omega\right) .
$$




\section{References}

Bojarski, N. N.: Generalized reaction principles and reciprocity theorems for the wave equations, and the relationship between the timeadvanced and time-retarded fields, J. Acoust. Soc. Am., 74, 281-285, 1983.

de Hoop, A. T.: Time-domain reciprocity theorems for acoustic wave fields in fluids with relaxation, J. Acoust. Soc. Am., 84, 1877-1882, 1988.

Fokkema, J. T. and van den Berg, P. M.: Seismic applications of acoustic reciprocity, Elsevier, Amsterdam, 1993.

Oristaglio, M. L.: An inverse scattering formula that uses all the data, Inverse Probl., 5, 1097-1105, 1989.

Porter, R. P.: Diffraction-limited, scalar image formation with holograms of arbitrary shape, J. Opt. Soc. Am., 60, 1051-1059, 1970.

Van Manen, D.-J., Robertsson, J. O. A., and Curtis, A.: Modeling of wave propagation in inhomogeneous media, Phys. Rev. Lett., 94, 164301, https://doi.org/10.1103/PhysRevLett.94.164301, 2005.

Wapenaar, C. P. A.: One-way representations of seismic data, Geophys. J. Int., 127, 178-188, 1996.

Wapenaar, C. P. A. and Berkhout, A. J.: Elastic wave field extrapolation, Elsevier, Amsterdam, 1989.

Wapenaar, K.: Retrieving the elastodynamic Green's function of an arbitrary inhomogeneous medium by cross correlation, Phys. Rev. Lett., 93, 254301, https://doi.org/10.1103/PhysRevLett.93.254301, 2004.

Wapenaar, K., van der Neut, J., Ruigrok, E., Draganov, D., Hunziker, J., Slob, E., Thorbecke, J., and Snieder, R.: Seismic interferometry by crosscorrelation and by multidimensional deconvolution: a systematic comparison, Geophys. J. Int., 185, 1335-1364, 2011.

Wapenaar, K., Thorbecke, J., van der Neut, J., Broggini, F., Slob, E., and Snieder, R.: Marchenko imaging, Geophysics, 79, WA39-WA57, 2014.

Wapenaar, K., Brackenhoff, J., and Thorbecke, J.: Green's theorem in seismic imaging across the scales, Solid Earth, 10, 517-536, 2019.

Zheng, Y., He, Y., and Fehler, M. C.: Crosscorrelation kernels in acoustic Green's function retrieval by wavefield correlation for point sources on a plane and a sphere, Geophys. J. Int., 184, 853-859, 2011. 